N. Toda

Nagoya Math. J.

Vol. 91 (1983), 37-47

\title{
ON THE FUNDAMENTAL INEQUALITY FOR DEGENERATE SYSTEMS OF ENTIRE FUNCTIONS
}

\section{Dedicated to Professor H. Ohtsuka on the occasion of his sixtieth birthday}

\section{NOBUSHIGE TODA}

\section{$\S 1$. Introduction}

Let $f=\left(f_{0}, f_{1}, \cdots, f_{n}\right)(n \geqq 1)$ be a transcendental system in $|z|<\infty$. That is, $f_{0}, f_{1}, \cdots, f_{n}$ are entire functions without common zeros and the characteristic function of $f$ defined by H. Cartan ([1]):

$$
T(r, f)=\frac{1}{2 \pi} \int_{0}^{2 \pi} U\left(r e^{i \theta}\right) d \theta-U(0)
$$

where

$$
U(z)=\max _{0 \leqq j \leqq n} \log \left|f_{j}(z)\right|
$$

satisfies the condition

$$
\lim _{r \rightarrow \infty} \frac{T(r, f)}{\log r}=\infty
$$

Let $X$ be a set of linear combinations $(\not \equiv 0)$ of $f_{0}, f_{1}, \cdots, f_{n}$ with coefficients in $C$ in general position; that is, for any $n+1$ elements

$$
a_{0 j} f_{0}+a_{1 j} f_{1}+\cdots+a_{n j} f_{n} \quad(j=1, \cdots, n+1)
$$

in $X, n+1$ vectors $\left(a_{0 j}, a_{1 j}, \cdots, a_{n j}\right)$ are linearly independent, and

$$
\lambda=\operatorname{dim}\left\{\left(c_{0}, c_{1}, \cdots, c_{n}\right) \in C^{n+1} ; c_{0} f_{0}+c_{1} f_{1}+\cdots+c_{n} f_{n}=0\right\} .
$$

It is clear that $0 \leqq \lambda \leqq n-1$. We note that, for any $n+1$ elements $F_{0}, F_{1}, \cdots, F_{n}$ in $X$,

$$
\operatorname{dim}\left\{\left(c_{0}, c_{1}, \cdots, c_{n}\right) \in C^{n+1} ; c_{0} F_{0}+c_{1} F_{1}+\cdots+c_{n} F_{n}=0\right\}
$$

is also equal to $\lambda$. We say that the system $f$ is degenerate when $\lambda>0$.

About fifty years ago, H. Cartan ([1]) proved

Received September 18, 1981. 
Theorem A. When $\lambda=0$, for any $q$ combinations $F_{1}, \cdots, F_{q}$ in $X$,

$$
(q-n-1) T(r, f) \leqq \sum_{j=1}^{q} N_{n}\left(r, 0, F_{j}\right)+S(r),
$$

where $N_{n}\left(r, 0, F_{j}\right)=N_{n}\left(r, F_{j}\right)$ in [1] and

$$
S(r)=O(\log r)+O(\log T(r, f))
$$

as $r \rightarrow \infty$ except for a set of finite linear measure.

He also gave the following conjecture for $\lambda \geqq 1$ (originally in the case of algebroid functions).

Conjecture of Cartan. For any $q$ combinations $F_{1}, \cdots, F_{q}$ in $X$,

$$
(q .-n-\lambda-1) T(r, f) \leqq \sum_{j=1}^{q} N_{n-\lambda}\left(r, 0, F_{j}\right)+S(r)
$$

It is uncertain that this conjecture is true or not in general, except when $\lambda=n-1$ ([1], p. 18). However, it is known that this holds in some special cases. For example,

Theorem B. For any $n+\lambda+2$ combinations $F_{1}, \cdots, F_{n+\lambda+2}$ in $X$,

$$
T(r, f) \leqq \sum_{j=1}^{n+\lambda+2} N_{n-\lambda}\left(r, 0, F_{j}\right)+S(r)
$$

([5]).

This theorem shows that Cartan's conjecture holds when $q=n+\lambda+2$.

The purpose of this paper is to prove that the conjecture is true when $\lambda=1$. Besides, we shall give an improvement of a result of $B$. Shiffman ([3]).

We use the standard notation of the Nevanlinna theory (See [2]).

\section{§2. Lemmas}

Let $f, X$ and $\lambda$ be as in Section 1. In this section, we shall give some lemmas which will be used in Section 3.

Lemma 1. For $H_{1}, \cdots, H_{k}$ in $X(2 \leqq k \leqq n+1-\lambda)$,

$$
m\left(r,\left\|H_{1}, \cdots, H_{k}\right\| / H_{1} \cdots H_{k}\right)=S(r),
$$

where $\left\|H_{1}, \cdots, H_{k}\right\|$ means the Wronskian of $H_{1}, \cdots, H_{k}$ (See [1]).

Lemma 2. For $F_{1}, \cdots, F_{q}$ in $X(q \geqq n+1)$, let 


$$
v(z)=\max _{\left(\beta_{1}, \cdots, \beta_{q-n}\right)} \log \left|F_{\beta_{1}}(z) \cdots F_{\beta_{q-n}}(z)\right|,
$$

where $\beta_{1}, \cdots, \beta_{q-n}$ are mutually disjoint $q-n$ numbers from $\{1,2, \cdots, q\}$. Then,

$$
(q-n) T(r, f) \leqq \frac{1}{2 \pi} \int_{0}^{2 \pi} v\left(r e^{i \theta}\right) d \theta+O(1)
$$

(See [4], Lemma 3).

Lemma 3. For any $G_{1}, \cdots, G_{q}$ in $X(q \geqq n+1)$, put

$$
u(z)=\min _{j_{1}<\cdots<j_{n+1-2}} \log \left|G_{j_{1}}(z) \cdots G_{j_{n+1-2}}(z)\right|,
$$

where $G_{j_{1}}, \cdots, G_{j_{n+1-\lambda}}$ are linearly independent and in $\left\{G_{j}\right\}$. Then,

$$
-S(r) \leqq \frac{1}{2 \pi} \int_{0}^{2 \pi} u\left(r e^{i \theta}\right) d \theta
$$

Proof. We suppose without loss of generality that $f_{0}, f_{1}, \cdots, f_{n-\lambda}$ are linearly independent. For an arbitrarily fixed $z=r e^{i \theta}$, we may suppose that

$$
\left|G_{1}(z)\right| \leqq\left|G_{2}(z)\right| \leqq \cdots \leqq\left|G_{q}(z)\right|
$$

for brevity. Then, there are $G_{j_{1}}, \cdots, G_{j_{n+1-\lambda}}\left(1 \leqq j_{1}<\cdots<j_{n+1-\lambda} \leqq n+1\right)$ which are linearly independent and satisfy

$$
u(z)=\log \left|G_{j_{1}}(z) \cdots G_{j_{n+1-\lambda}}(z)\right| .
$$

As

$$
\left\|G_{j_{1}}, \cdots, G_{j_{n+1-\lambda}}\right\|=c\left\|f_{0}, \cdots, f_{n-\lambda}\right\| \quad(c \neq 0, \text { constant })
$$

we have

$$
\frac{G_{j_{1}} \cdots G_{j_{n+1-\lambda}}}{\left\|G_{j_{1}}, \cdots, G_{j_{n+1-\lambda}}\right\|}=\frac{G_{j_{1}} \cdots G_{j_{n+1-\lambda}}}{c\left\|f_{0}, \cdots, f_{n-2}\right\|}
$$

so that

$$
\log \left|\left\|f_{0}, \cdots, f_{n-\lambda}\right\|\right| \leqq u(z)+\sum_{j_{1}, \cdots, j_{n+1-\lambda}}^{q} \log ^{+}\left|\frac{\left\|G_{j_{1}}, \cdots, G_{j_{n+1-\lambda}}\right\|}{G_{j_{1}} \cdots G_{j_{n+1-\lambda}}}\right|+O(1),
$$

where $O(1)$ is a constant dependent only on $G_{1}, \cdots, G_{q}$. This inequality holds for any $z$. Integrating with respect to $\theta$ from 0 to $2 \pi$ and dividing by $2 \pi$, we obtain 


$$
N\left(r, 0,\left\|f_{0}, \cdots, f_{n-\lambda}\right\|\right) \leqq \frac{1}{2 \pi} \int_{0}^{2 \pi} u\left(r e^{i \theta}\right) d \theta+S(r)
$$

which includes the desired inequality.

According to B. Shiffman ([3]), we let $\mathscr{E}_{\rho}$ denote the ring of entire functions of the form

$$
g(z)=\sum_{k=1}^{p} \phi_{k}(z) \exp P_{k}(z)
$$

where the $P_{k}$ are polynomials of degree at most $\rho$ and the $\phi_{k}$ are meromorphic functions in $|z|<\infty$ such that

$$
T\left(r, \phi_{k}\right)=o\left(r^{\rho}\right) \quad(r \rightarrow \infty) .
$$

Moreover, according to Definition 1 ([3]), we say that a system $f=$ $\left(f_{0}, \cdots, f_{n}\right)$ is of special exponential type of order $\rho(0<\rho<\infty)$ if

$$
c_{1} r^{\rho}<T(r, f)<c_{2} r^{\rho} \quad \text { as } r \rightarrow \infty,
$$

where $c_{1}$ and $c_{2}$ are positive constants, and if $f_{0}, \cdots, f_{n}$ belong to $\mathscr{E}_{\rho}$.

Lemma 4. Let $h=\left(h_{1}, \cdots, h_{N}\right)$ be of special exponential type of order $\rho$ such that $h_{j} \not \equiv 0$ for $1 \leqq j \leqq N$. Then,

$$
\frac{1}{2 \pi} \int_{0}^{2 \pi} \log \sum_{j=1}^{N}\left(1 /\left|h_{j}\left(r e^{i \theta}\right)\right|\right) d \theta \leqq \frac{1}{2 \pi} \int_{0}^{2 \pi} \log \sum_{j=1}^{N}\left|h_{j}\left(r e^{i \theta}\right)\right| d \theta+o\left(r^{\rho}\right)
$$

as $r \rightarrow \infty$ ([3], Lemma 2).

\section{§3. Theorems}

Let $f, X$ and $\lambda$ be as in Section 1 .

TheOREM 1 . When $\lambda=1$, for any $q(q \geqq n+2)$ combinations $F_{1}, \cdots, F_{q}$ in $X$,

$$
(q-n-2) T(r, f) \leqq \sum_{j=1}^{q} N_{n-1}\left(r, 0, F_{j}\right)+S(r) .
$$

Proof. We may suppose that $f_{1}, \cdots, f_{n}$ are linearly independent without loss of generality since $\lambda=1$. Now, there exists an integer $k$ such that any $k$ elements in $X_{0}=\left\{F_{j}\right\}_{j=1}^{q}$ are linearly independent, but some $k+1$ elements in $X_{0}$ are linearly dependent. It is clear that $1 \leqq k \leqq n$. For an arbitrarily fixed $z=r e^{i \theta}(r>0)$, let $K_{1}, \cdots, K_{n+1}$ be $n+1$ elements of $X_{0}$ such that $\left|K_{1}(z)\right|, \cdots,\left|K_{n+1}(z)\right|$ are the smallest $n+1$ elements of 
$\left\{\left|F_{1}(z)\right|, \cdots,\left|F_{q}(z)\right|\right\} . \quad$ As $\lambda=1$, we suppose without loss of generality that $K_{1}, \cdots, K_{n}$ are linearly independent and

$$
K_{n+1}=\alpha_{1} K_{1}+\cdots+\alpha_{m} K_{m} \quad\left(m \geqq k, \alpha_{1} \cdots \alpha_{m} \neq 0\right) .
$$

Put

$$
W_{0}=\left\|K_{1}, \cdots, K_{n}\right\| \prod_{j=1}^{k}\left\|K_{1}, \cdots, K_{j-1}, K_{n+1}, K_{j+1}, \cdots, K_{n}\right\|,
$$

then, $W_{0} \not \equiv 0$ and in $W_{0}, K_{1}, \cdots, K_{k}$ and $K_{n+1}$ appear $k$ times and $K_{k+1}, \cdots$. $K_{n}$ appear $k+1$ times. Since

$$
\left\|K_{1}, \cdots, K_{n}\right\|=c_{1}\left\|f_{1}, \cdots, f_{n}\right\|,
$$

where $c_{1}$ is a constant $(\neq 0)$, we have the equality

$$
\frac{\left(F_{1} \cdots F_{q}\right)^{k}}{W_{0}}=\frac{\left(F_{1} \cdots F_{q}\right)^{k}}{c\left\|f_{1}, \cdots, f_{n}\right\|^{k+1}} \quad\left(c=c_{1}^{k+1} \alpha_{1} \cdots \alpha_{k}\right)
$$

so that we obtain the following inequality as usual (cf. [1], [4]):

$$
\begin{aligned}
k(q-n-1) U(z) \leqq & k \sum_{j=1}^{q} \log \left|F_{j}(z)\right|+\sum_{j_{1}, \cdots, j_{n}=1}^{q} \log ^{+}\left|\frac{\left\|F_{j_{1}}, \cdots, F_{j_{n}}\right\|}{F_{j_{1}} \cdots F_{j_{n}}}\right| \\
& +(n-k) U(z)-(k+1) \log \left|\left\|f_{1}, \cdots, f_{n}\right\|\right|+O(1),
\end{aligned}
$$

where $O(1)$ is a constant depending only on $X_{0}$. This inequality holds for every $z$, so that, integrating with respect to $\theta$ from 0 to $2 \pi$ and dividing by $2 \pi$, we obtain

$$
\begin{aligned}
k(q-n-1) T(r, f) \leqq & k \sum_{j=1}^{q} N\left(r, 0, F_{j}\right)+(n-k) T(r, f) \\
& -(k+1) N\left(r, 0,\left\|f_{1}, \cdots, f_{n}\right\|\right)+S(r)
\end{aligned}
$$

by Lemma 1 ; that is,

$$
\begin{aligned}
(q- & n-1-(n-k) / k) T(r, f) \\
\leqq & \sum_{j=1}^{q} N\left(r, 0, F_{j}\right)-(1+1 / k) N\left(r, 0,\left\|f_{1}, \cdots, f_{n}\right\|\right) \\
& \quad+S(r) \leqq \sum_{j=1}^{q} N_{n-1}\left(r, 0, F_{j}\right)+S(r) .
\end{aligned}
$$

We have the last inequality by calculating the multiplicity of zero at $z$ of the righthand side of (1) as in the case of the fundamental theorem of Cartan ([1], p. 14).

I. Therefore, when $(n-k) / k \leqq 1$, that is, $n / 2 \leqq k$, we have the theorem. 
II. Next, we prove this theorem when $1 \leqq k<n / 2$. To begin with, we note that there exists an element $G$ in $X_{0}$ such that any $n-k$ elements in $X_{0}-\{G\}$ are linearly independent. Indeed, let $G, H_{1}, \cdots, H_{k}$ be $k+1$ elements in $X_{0}$ which are linearly dependent, then $G$ may be represented by $H_{1}, \cdots, H_{k}$ :

$$
G=d_{1} H_{1}+\cdots+d_{k} H_{k} \quad\left(d_{1} \cdots d_{k} \neq 0\right)
$$

because of the definition of the number $k$. If there exist $I_{1}, \cdots, I_{n-k}$ in $X_{0}-\{G\}$ which are linearly dependent, there are at least two distinct linear relations among $G, H_{1}, \cdots, H_{k}, I_{1}, \cdots, I_{n-k}$. This is a contradiction to the hypothesis of $\lambda=1$. Let

$$
X_{0}-\{G\}=\left\{G_{1}, G_{2}, \cdots, G_{q-1}\right\} .
$$

For a fixed $z=r e^{i \theta}(\neq 0)$, we may suppose for brevity that

$$
\left|G_{j}(z)\right| \leqq\left|G_{n+1}(z)\right| \leqq \cdots \leqq\left|G_{q-1}(z)\right| \quad(j=1, \cdots, n) .
$$

We consider the following two cases.

(i) The case when $G_{1}, \cdots, G_{n}$ are linearly dependent.

Let, for example, without loss of generality

$$
G_{n}=\beta_{1} G_{1}+\cdots+\beta_{\nu} G_{\nu} \quad\left(\beta_{1} \cdots \beta_{\nu} \neq 0\right)
$$

then $\nu \geqq n-k$ and $G_{1}, \cdots, G_{n-1}, G$ are linearly independent. Consider the following product

$$
W_{1}=\left\|G_{1}, \cdots, G_{n-1}, G\right\| \prod_{j=1}^{n-k}\left\|G_{1}, \cdots, G_{j-1}, G_{n}, G_{j+1}, \cdots, G_{n-1}, G\right\| .
$$

Then, $W_{1} \neq \equiv$ and in $W_{1}, G_{1}, \cdots, G_{n-k}$ appear $n-k$ times and $G_{n+1-k}, \cdots$, $G_{n-1}, G$ appear $n+1-k$ times. As in (1), we obtain

$$
\frac{\left(G_{1} \cdots G_{q-1}\right)^{n-k}}{W_{1}}=\frac{\left(G_{1} \cdots G_{q-1}\right)^{n-k}}{c_{1}\left\|f_{1}, \cdots, f_{n}\right\|^{n+1-k}} \quad\left(c_{1} \neq 0, \text { constant }\right)
$$

so that we have the following inequality:

$$
\begin{aligned}
(n-k) v_{1}(z) \leqq & (n-k) \sum_{j=1}^{q-1} \log \left|G_{j}(z)\right|+(n-k) \log |G(z)|+k U(z) \\
& +\sum_{j_{1}, \cdots, j_{n}=1}^{q} \log ^{+}\left|\frac{\left\|F_{j_{1}}, \cdots, F_{j_{n}}\right\|}{F_{j_{1}} \cdots F_{j_{n}}}\right| \\
& -(n+1-k) \log \left|\left\|f_{1}, \cdots, f_{n}\right\|\right|+O(1),
\end{aligned}
$$


where $v_{1}(z)$ is equal to $v(z)$ given in Lemma 2 for $G_{1}, \cdots, G_{q-1}$ and $O(1)$ is dependent only on $X_{0}$.

As

$$
\log |G(z)| \leqq U(z)+O(1)
$$

and $n-k>k$, we have

$$
(n-k) \log |G(z)|+k U(z) \leqq k \log |G(z)|+(n-k) U(z)+O(1) .
$$

Therefore,

$$
\begin{aligned}
(n-k) v_{1}(z) \leqq & (n-k) \sum_{j=1}^{q-1} \log \left|G_{j}(z)\right|+k \log |G(z)|+(n-k) U(z) \\
& -(n+1-k) \log \left|\left\|f_{1}, \cdots, f_{n}\right\|\right| \\
& +\sum_{j_{1}, \cdots, j_{n}=1}^{q} \log ^{+}\left|\frac{\left\|F_{j_{1}}, \cdots, F_{j_{n}}\right\|}{F_{j_{1}} \cdots F_{j_{n}}}\right|+O(1) .
\end{aligned}
$$

(ii) The case when $G_{1}, \cdots, G_{n}$ are linearly independent.

In this case $G$ can be represented by $G_{1}, \cdots, G_{n}$; that is, without loss of generality we may write

$$
G=\gamma_{1} G_{1}+\cdots+\gamma_{\mu} G_{\mu} \quad\left(\mu \geqq k, \gamma_{1} \cdots \gamma_{\mu} \neq 0\right) .
$$

Consider the following product

$$
W_{2}=\left\|G_{1}, \cdots, G_{n}\right\|^{n+1-2 k} \prod_{j=1}^{k}\left\|G_{1}, \cdots, G_{j-1}, G, G_{j+1}, \cdots, G_{n}\right\| \cdot
$$

Then, $W_{2} \not \equiv 0$ and in $W_{2}, G_{1}, \cdots, G_{k}$ appear $n-k$ times, $G_{k+1}, \cdots, G_{n}$ appear $n+1-k$ times and $G$ appears $k$ times. As in (3), it holds the following equality:

$$
\frac{\left(G_{1} \cdots G_{q-1}\right)^{n-k}}{W_{2}}=\frac{\left(G_{1} \cdots G_{q-1}\right)^{n-k}}{c_{2}\left\|f_{1}, \cdots, f_{n}\right\|^{n+1-k}} \quad\left(c_{2} \neq 0, \text { constant }\right)
$$

from which we obtain the following inequality:

$$
\begin{aligned}
(n-k) v_{1}(z) \leqq & (n-k) \sum_{j=1}^{q-1} \log \left|G_{j}(z)\right|+k \log |G(z)|+(n-k) U(z) \\
& -(n+1-k) \log \left|\left\|f_{1}, \cdots, f_{n}\right\|\right| \\
& +\sum_{j_{1}, \cdots, j_{n}=1}^{q} \log ^{+}\left|\frac{\left\|F_{j_{1}}, \cdots, F_{j_{n}}\right\|}{F_{j_{1}} \cdots F_{j_{n}}}\right|+O(1) .
\end{aligned}
$$

In both cases (i) and (ii), we obtain the same inequality (4) or (6) which holds for any $z(\neq 0)$. Integrating the inequality with respect to $\theta$ from 
0 to $2 \pi$, dividing by $2 \pi$ and applying Lemmas 1 and 2 , we have

$$
\begin{aligned}
(n-k)(q-n-1) T(r, f) \leqq & (n-k) \sum_{j=1}^{q-1} N\left(r, 0, G_{j}\right)+k N(r, 0, G) \\
& +(n-k) T(r, f) \\
& -(n+1-k) N\left(r, 0,\left\|f_{1}, \cdots, f_{n}\right\|\right)+S(r),
\end{aligned}
$$

that is,

$$
\begin{aligned}
(q-n-2) T(r, f) \leqq & \sum_{j=1}^{q-1} N\left(r, 0, G_{j}\right)+k N(r, 0, G) /(n-k) \\
& -(1+1 /(n-k)) N\left(r, 0,\left\|f_{1}, \cdots, f_{n}\right\|\right) \\
& +S(r) \leqq \sum_{j=1}^{q} N_{n-1}\left(r, 0, F_{j}\right)+S(r) .
\end{aligned}
$$

We can easily prove the last inequality using the following inequality (8). Let $m_{j}$ be the multiplicity of zero of $G_{j}$ at $z(j=1, \cdots, q-1)$ and $m$ that of $G$ at $z$, then we obtain

$$
\begin{aligned}
& \text { the multiplicity of zero of } \frac{\left(G_{1} \cdots G_{q-1}\right)^{n-k} G^{k}}{\left\|f_{1}, \cdots, f_{n}\right\|^{n+1-k}} \\
& \leqq(n-k) \sum_{j=1}^{q-1} \min \left(m_{j}, n-1\right)+k \min (m, n-1)
\end{aligned}
$$

applying the method used in the proof of the fundamental theorem of Cartan ([1]) to

$$
\frac{\left(G_{1} \cdots G_{q-1}\right)^{n-k} G^{k}}{W_{j}}=\frac{\left(G_{1} \cdots G_{q-1}\right)^{n-k} G^{k}}{c_{j}\left\|f_{1}, \cdots, f_{n}\right\|^{n+1-k}} \quad(j=1 \text { or } 2) .
$$

Thus the proof of our theorem is complete.

Corollary 1. Under the same assumption as in Theorem 1,

$$
\sum_{F \in X} \delta(F) \leqq n+2
$$

If the equality holds in (9) and if $n$ is odd, there are at least two $F$ in $X$ for which $\delta(F)=1$. Here, $\delta(F)=1-\lim \sup _{r \rightarrow \infty} N(r, 0, F) / T(r, f)$.

Proof. We can prove easily (9) as usual. Now, suppose that $n$ is odd and

$$
\sum_{F \in X} \delta(F)=n+2 .
$$

In the sequel in this proof, we use the same notation as in the proof of 
Theorem 1. Let $\varepsilon$ be any positive number smaller than $1 / n$. Then, there are $F_{1}, \cdots, F_{q}$ in $X$ for which $\delta\left(F_{j}\right)>0(j=1, \cdots, q)$ and such that

$$
n+2-\varepsilon<\sum_{j=1}^{q} \delta\left(F_{j}\right) \text {. }
$$

Then for $X_{0}=\left\{F_{1}, \cdots, F_{q}\right\}, k<n / 2$. Because, if $k \geqq n / 2$, then $k \geqq(n+1) / 2$ since $n$ is odd and from (2) we have

$$
\sum_{j=1}^{q} \delta\left(F_{j}\right) \leqq n+1+(n-k) / k \leqq n+2-2 /(n+2),
$$

which contradicts (10).

There are $G, H_{1}, \cdots, H_{k}$ in $X_{0}$ such that

$$
G=d_{1} H_{1}+\cdots+d_{k} H_{k} \quad\left(d_{1} \cdots d_{k} \neq 0\right)
$$

as in II. Suppose

$$
\delta=\min \left\{\delta(G), \delta\left(H_{1}\right), \cdots, \delta\left(H_{k}\right)\right\}<1
$$

and let $\varepsilon^{\prime}$ be any positive number smaller than $(1-\delta) / n$. Let $X_{1}$ be a finite subset of $X$ which contains $X_{0}$ such that

$$
n+2-\varepsilon^{\prime}<\sum_{F \in X_{1}} \delta(F) .
$$

Then any $k+1$ elements in $X_{1}$ which are not in coincidence with $\left\{G, H_{1}, \cdots, H_{k}\right\}$ are linearly independent as $k \leqq(n-1) / 2$ and $\lambda=1$. Indeed, if there are $k+1$ elements $I_{1}, \cdots, I_{k+1}$ in $X_{1}$ which are linearly dependent and don't coincide with $\left\{G, H_{1}, \cdots, H_{k}\right\}$, then $2(k+1) \leqq n+1$ and there are at least two linearly independent linear relations among $G, H_{1}, \cdots, H_{k}$, $I_{1}, \cdots, I_{k+1}$. That is, $\lambda \geqq 2$, which is a contradiction

Now, as is easily seen, we can use any one of $\left\{H_{j}\right\}_{j=1}^{k}$ instead of $G$ in II so that from the first inequality in (7), we have

$$
\sum_{F \in X_{1}} \delta(F) \leqq n+1+(n-2 k)(1-\delta) /(n-k),
$$

which contradicts (11). This shows that $\delta$ must be equal to 1 and so

$$
\delta(G)=\delta\left(H_{1}\right)=\cdots=\delta\left(H_{k}\right)=1 .
$$

This completes the proof.

THEOREM 2. Suppose that $f$ is of special exponential type of order $\rho$. Then for any $F_{1}, \cdots, F_{q}$ in $X$, 


$$
(q-n-\lambda-1) T(r, f) \leqq \sum_{j=1}^{q} N\left(r, 0, F_{j}\right)+o(T(r, f))+S(r)
$$

Proof. We have only to prove this theorem when $q \geqq n+\lambda+2$. Let $h_{1}=F_{1} F_{2} \cdots F_{\lambda}, h_{2}=F_{1} \cdots F_{\lambda-1} F_{\lambda+1}, \cdots, h_{N}=F_{q+1-\lambda} \cdots F_{q}\left(N=\left(\begin{array}{l}q \\ 2\end{array}\right)\right)$. Then, $h_{j} \not \equiv 0$ and $h=\left(h_{1}, \cdots, h_{N}\right)$ is a system of special exponential type of order $\rho$. Now, for an arbitrarily fixed $z=r e^{i \theta}(\neq 0)$, we suppose without loss of generality that

$$
\left|F_{1}(z)\right| \leqq\left|F_{2}(z)\right| \leqq \cdots \leqq\left|F_{q}(z)\right|
$$

Then

$$
u(z)+(q-n-1) U(z) \leqq \sum_{j=1}^{q} \log \left|F_{j}(z)\right|+\log \sum_{j=1}^{N}\left|1 / h_{j}(z)\right|+O(1),
$$

where $O(1)$ is dependent only on $\left\{F_{j}\right\}_{j=1}^{q}$, so that we have by Lemmas 3 and 4

$$
\begin{aligned}
(q-n-1) T(r, f) \leqq & \sum_{j=1}^{q} N\left(r, 0, F_{j}\right) \\
& +\frac{1}{2 \pi} \int_{0}^{2 \pi} \log \sum_{j=1}^{N}\left|h_{j}\left(r e^{i \theta}\right)\right| d \theta+o\left(r^{\rho}\right)+S(r) .
\end{aligned}
$$

Here we use the following inequalities

$$
\left|h_{j}(z)\right| \leqq a_{j} \exp \lambda U(z) \quad(j=1, \cdots, N),
$$

where $a_{j}$ are constants. These are true because

$$
\left|F_{\nu}(z)\right| \leqq b_{\nu} \max _{0 \leqq j \leqq n}\left|f_{j}(z)\right| \quad(\nu=1, \cdots, q)
$$

and

$$
\left|h_{j}(z)\right| \leqq a_{j}\left(\max _{0 \leqq j \leqq n}\left|f_{j}(z)\right|\right)^{\lambda}=a_{j} \exp \lambda U(z) \quad(j=1, \cdots, N)
$$

That is, we obtain

$$
(q-n-\lambda-1) T(r, f) \leqq \sum_{j=1}^{q} N\left(r, 0, F_{j}\right)+o\left(r^{\rho}\right)+S(r),
$$

which is the desired inequality.

CoRollary 2. Under the same assumption of Theorem 2,

$$
\sum_{F \in X} \delta(F) \leqq n+\lambda+1
$$




\section{REFERENCES}

[1] H. Cartan, Sur les zéros des combinaisons linéaires de $p$ fonctions holomorphes données, Mathematica, 7 (1933), 5-31.

[2] W. K. Hayman, Meromorphic functions, Clarendon Press, Oxford, 1964.

[3] B. Shiffman, On holomorphic curves and meromorphic maps in projective space, Indiana Univ. Math. J., 28 (1979), 627-641.

[4:] N. Toda, Sur quelques combinaisons linéaires exceptionnelles au sens de Nevanlinna, Tôhoku Math. J., 23 (1971), 67-95.

[5] - Sur l'inégalité fondamentale de H. Cartan pour les systèmes de fonctions entières, Nagoya Math. J., 83 (1981), 5-14.

Department of Mathematics

Nagoya Institute of Technology

Showa-ku, Nagoya 466

Japan 